COMO CITAR ESTE ARTÍCULO:

Urbina, F.J. y Contreras, F. (2019). Derecho y ordenamiento de la conducta. Revista Jurídicas, 16 (2), 108-125.

DOI: 10.17151/jurid.2019.16.2.8.

Recibido el 3 de mayo de 2018

Aprobado el 26 de marzo de 2019

\section{Derecho y ordenamiento de la conducta*}

\author{
Francisco J. Urbina** \\ Fernando Contreras***
}

\section{RESUMEN}

En este artículo se explora la forma en que el derecho ordena la conducta humana. El argumento se construye sobre el supuesto elemental según el cual el derecho aspira a ordenar la conducta de las personas, y se inserta en la teorización de Lon Fuller sobre los desiderata del Estado de Derecho. El objetivo del artículo es presentar, justificar y analizar la tesis de que el derecho ordena la conducta de manera principalmente directa, es decir, no mediante manipulación o estímulos sino significando un orden deseado. Siguiendo la metodología de Fuller, consistente en identificar las condiciones para que el derecho genere orden, se concluye que la ordenación directa de la conducta es más eficaz y, además, consistente con la dignidad moral de aquellos sujetos al derecho. Así, se propone un desideratum adicional a la articulación de Fuller: que el derecho ordene la conducta humana de manera directa.
Palabras clave: teoría del derecho, ordenamiento de la conducta, razón práctica, Lon Fuller.

\footnotetext{
* Este trabajo es parte del proyecto CONICYT-FONDECYT Iniciación 11150872 del cual Francisco Urbina es el investigador principal.

** Doctor en Derecho, Universidad de Oxford. Profesor Asistente, Pontificia Universidad Católica de Chile, Santiago, Chile. E-mail: fjurbina@uc.cl.

Google Scholar. ORCID: 0000-0001-5427-2704.

*** Licenciado en Derecho, Pontificia Universidad Católica de Chile. Profesor Instructor Adjunto, Pontificia Universidad Católica de Chile. Santiago, Chile. E-mail: ftcontre@uc.cl.

Google Scholar. ORCID: 0000-0002-9660-2262.
} 


\section{Law and ordering of behaviour}

\section{ABSTRACT}

This article explores the way in which law orders human behaviour. The argument builds on the basic assumption that law aims to order human conduct, and is inserted in Lon Fuller's desiderata theory of the Rule of Law. The purpose of the article is to present, justify and analyze the thesis that law mainly orders human behaviour "straightforwardly", that is, not through manipulation or stimuli. Following Fuller's methodology, which seeks to identify the requirements law must meet to produce order, it concludes that the straightforwardly ordering of human conduct is both more effective and consistent with the moral dignity of those subject to the law. Thus, it proposes an additional desideratum to Fuller's theory: that law must order human behaviour in a straightforward way.

KEY WORDS: jurisprudence, order of human behaviour, practical reason, Lon Fuller. 


\section{Introducción}

La idea de que el derecho ${ }^{1}$ pretende ordenar la conducta humana es pacífica en la literatura. Así, distintas escuelas iusfilosóficas, opuestas en otros sentidos, coinciden en entender que, a pesar de cualquiera otra característica que pueda tener, el derecho es básicamente un orden de la conducta humana. Afirmar que el derecho pretende y es capaz de ordenar la conducta humana es afirmar algo muy elemental sobre el derecho. Las teorías sobre el derecho, en general, dejan este supuesto elemental en paz, y se abocan a explorar asuntos que exigen una visión más elaborada, tales como la forma en que se estructura el sistema jurídico ${ }^{2}$, la fuerza normativa específica de ciertas partes del derecho (por ejemplo, los derechos; Dworkin, 1978; Alexy, 2007), o la forma en que el derecho positivo y el razonamiento práctico debieran interactuar en la interpretación jurídica (Dworkin, 1986).

Es en la influyente articulación de las exigencias [desiderata] del Estado de Derecho que realiza Lon Fuller donde se aprecia de forma más notoria la relevancia de este supuesto elemental. Para Fuller, el derecho consiste en "la empresa de ordenar la conducta humana al gobierno de las reglas". Sus famosos desiderata son ciertas exigencias formales que un sistema jurídico debe cumplir para poder efectivamente ordenar la conducta humana (Fuller, 1969, p. 53, 96-97, 106 y, en general, el capítulo 2). A todas estas exigencias subyace el supuesto básico de que el derecho ha de ordenar la conducta.

En este trabajo pretendemos avanzar en una caracterización más detallada de la forma general en que el derecho ordena la conducta humana. Nos interesa destacar un aspecto de la forma en que el derecho ordena la conducta que no ha sido tematizado directamente y que, sin embargo, es crucial en su comprensión general. Nuestra tesis es que el derecho ordena la conducta humana de forma primordialmente "directa". Es decir, no mediante manipulación o estímulos sino comunicando un determinado orden al cual se espera y desea que los sujetos del derecho se conformen.

El desarrollo de nuestra tesis se hará por medio del método siguiente. Exploraremos el asunto enmarcándolo en la teoría del derecho que más explícitamente se ha

\footnotetext{
1 Este trabajo se preocupa fundamentalmente del derecho positivo, aunque entendiéndolo en sentido amplio, incluyendo obras (como tratados o recopilaciones) que no emanan de órganos con potestades jurídicas y que, sin embargo, alcanzan el estatus de canónicas en una determinada comunidad jurídica donde, por lo tanto, las categorías jurídicas contenidas en ellas pasan a ser autoritativas para esa comunidad jurídica (para un estudio de obras de este tipo, centrado en la codificación, véase Jansen (2010)). Pero al hablar del derecho nuestra referencia es el derecho artificial, técnico, producido por el hombre, y no exigencias de la razón práctica cuya existencia, contenido y legitimidad son independientes de la adopción o promulgación humana. Utilizamos el término "derecho" para referirnos a este objeto de estudio.

${ }^{2}$ Por ejemplo, en el orden jerárquico de normas que se dotan unas a otras de validez asociado a la "construcción escalonada del orden jurídico" descrito por Kelsen en su "dinámica jurídica" (Kelsen, 1979, p. 201-284), o en la interacción de reglas primarias y secundarias con que Hart caracteriza un sistema jurídico desarrollado (Hart, 2012, p. 79-99).
} 
interrogado por la forma en que el derecho ordena la conducta humana, la teoría de los desiderata de Lon Fuller. En este marco, lo que orienta este trabajo es determinar si la pretensión de que el derecho ordene la conducta humana exige que la dirección que el derecho otorga sea "directa". En otras palabras, si la dirección directa constituye o no un desideratum.

No hay razón para dejar al lector en suspenso: la respuesta es afirmativa. Proponemos, por consiguiente, un desideratum adicional a los formulados por Fuller: que las reglas deben aspirar a ordenar la conducta humana de forma directa.

El argumento se desarrollará de la siguiente manera. En la primera sección explicaremos el supuesto elemental, así como la teoría de los desiderata de Lon Fuller, a cuya elaboración esperamos contribuir. A continuación, en la segunda la más extensa-, introduciremos la distinción entre formas directas e indirectas de ordenar la conducta humana, y argumentaremos que es deseable que el derecho corresponda primordialmente a las primeras. Esto quiere decir, en otras palabras, que es un desideratum (en los términos de Fuller) del derecho que las reglas jurídicas ordenen la conducta humana de forma directa. En la tercera sección ofreceremos algunas aclaraciones respecto del alcance e implicancias de este desideratum.

\section{Un supuesto elemental: el derecho pretende ordenar la conducta humana}

El derecho se ocupa de la conducta humana, aspirando a ordenarla de cierto modo acorde a sus dictados. Esta parece ser una idea ampliamente compartida por las distintas teorías del derecho. Así lo entiende Fuller, como hemos enunciado. En el mismo sentido, Kelsen (1949) abre la parte sustantiva de su Teoría general del derecho y del Estado afirmando: "El derecho es un orden de la conducta humana" (p. 3). Este orden, como todo orden social, tendría la función de "provocar cierta conducta recíproca entre los seres humanos" (Kelsen, 1949, p. 15; algo también compartido con Fuller, véase, p. 39-40, 48, 61-62), y su característica específica sería la de un orden coactivo basado en la "técnica social que consiste en provocar la conducta socialmente deseada a través de la amenaza de una medida coercitiva que debe aplicarse en caso de un comportamiento contrario" (Kelsen, 1949, p. 20). De forma similar, para Austin el derecho consiste en el conjunto de "leyes o reglas dadas por hombres a hombres [...] establecidas por 'superiores políticos'" (Austin, 1998, p. 11), entendiendo que toda "ley o regla [...] es una orden" (Austin, 1998, p. 13), y que el sentido de una "orden" es ordenar la conducta de otra persona (Austin, 1998, p. 13-14)3. De acuerdo con una reciente teoría del derecho, la de Scott Shapiro, el derecho es concebido en términos de planes. Así, para Shapiro, "el

\footnotetext{
${ }^{3}$ Para Austin, la idea de "orden" implica la "expresión o intimación de un deseo de que [alguien] realice o se abstenga de realizar un acto", unido, como es sabido, a la amenaza de "aplicar un mal" en el caso de que esa persona "no cumpla" con el deseo (Austin, 1998, p. 13-14). Salvo que se indique lo contrario, todas las traducciones son propias.
} 
ejercicio de la autoridad jurídica es una actividad de planificación social" (Shapiro, 2011, p. 195). La planificación en el derecho tiene los mismos "objetivos básicos" que en otras áreas de la vida social: "guiar, organizar y monitorear la conducta de los individuos y grupos" (Shapiro, 2011, p. 200). Es decir, ordenar la conducta humana. De similar modo, para John Finnis el sistema jurídico de una comunidad, en su caso central, "pretende tener autoridad para dar dirección comprehensiva y suprema a la conducta humana en esa comunidad" (Finnis, 2011a, p. 260). Para lograr esto, el sistema jurídico debe satisfacer las exigencias que Fuller identificó y etiquetó como desiderata del Estado de Derecho (Finnis, 2011a, p. 270-271) ${ }^{4}$.

\section{La remisión de Finnis a Fuller no es casual. La teoría de Fuller es el referente obligado en este tema, toda vez que contiene la versión canónica de dichos desiderata ${ }^{5}$ En su influyente libro The Morality of Law, Fuller presenta una teoría del derecho en cuyo núcleo se encuentran una serie de exigencias (desiderata, término latino}

\footnotetext{
${ }^{4}$ Una objeción tan fácil como reiterada en algunos medios académicos a prácticamente cualquier tesis constructiva en teoría general del derecho es: “¿cómo se hace Ud. cargo del realismo jurídico americano?" El supuesto es que el realista jurídico, al negar la posibilidad de que las normas jurídicas puedan controlar la acción humana, boicotea gran parte de lo que se pueda decir a nivel teórico sobre cómo opera el derecho (incluyendo lo que se pueda decir sobre cómo el derecho ordena la conducta humana, pues no la ordenaría), y sólo quedaría observar empíricamente la práctica de quienes lo aplican. Pero esto descansa sobre una caricatura. Gran parte de la crítica de los llamados realistas jurídicos está dirigida a los excesos de la "jurisprudencia clásica" norteamericana que los antecedió y, en particular, contra un optimismo excesivo en la determinación y coherencia interna del derecho (Fisher et al., 1993, p. xi-xii) (para una exposición más detallada de la jurisprudencia clásica, véase Grey (1983)). Su escepticismo era considerable para la época, pero no es de la radicalidad que se les supone. Así, por ejemplo, en los trabajos tardíos de quien es posiblemente el realista jurídico más relevante — Karl Llewellyn—, se enfatiza que si bien el derecho no es tan determinado ni tan capaz de controlar la conducta de los agentes del derecho como pensaban los juristas norteamericanos de la generación precedente a la suya (la jurisprudencia clásica), es excesivo decir que no lo puede hacer en absoluto, afirmando que "[e]l rol de las reglas es controlar, guiar, y unificar la conducta de los agentes del derecho" (Llewellyn, 2011, p. 48-49). Ya antes había sostenido algo similar en los agradecimientos a la segunda edición de 1951 de su libro más famoso, The Bramble Bush, en el que comentaba la frase más analizada y criticada de la edición original de 1930 ("lo que estos agentes del derecho hacen respecto de las disputas es, en mi parecer, el derecho mismo." (Llewellyn, 2008, p. 5)). Sostuvo que la expresión era "poco feliz cuando no se desarrollaba más plenamente" y que "es claro que un rol del derecho es controlar a los agentes del derecho en parte, y guiarlos incluso en asuntos en los que un control completo no es posible ni deseable" (Llewellyn, 2008, p. xxviii). Se puede decir algo semejante del movimiento "Critical Legal Studies" y la tesis de la radical indeterminación del derecho. Dicha tesis no es representativa de todo el movimiento ni tal vez de sus cultores centrales. Como sostiene, en una mirada panorámica sobre el desarrollo del movimiento, uno de sus exponentes más relevantes: "la enseñanza de la radical indeterminación llevó a sus defensores a un desierto y los abandonó ahí sin recursos ni perspectivas" (Unger, 2015, p. 26). "El deconstruccionismo, centrado en la radicalización de la indeterminación del razonamiento jurídico, redujo el derecho a algo que nunca ha sido: una serie de oportunidades para la manipulación retórica, limitadas solo por la influencia de una ideología o forma de consciencia afianzada, compartida, y no reconocida. Demostró ser un camino sin salida intelectual y política" (Unger, 2015, p. 44).

Por supuesto, el punto no es que todas las teorías sobre el derecho compartan el supuesto esencial sobre el que versa este trabajo (que el derecho es una forma de ordenar la conducta humana), sino que lo hace un abanico suficientemente amplio como para justificar su tratamiento como un supuesto elemental ampliamente compartido. Y claro, el análisis de este artículo le parecerá que no tiene sentido al escéptico jurídico radical, así como le parecerá sin sentido buena parte de la teoría del derecho y todo lo que se escribe sobre dogmática jurídica; y lo mismo al escéptico radical moral le parecerá sin sentido buena parte de lo que se escribe en la filosofía práctica, así como al escéptico radical metafísico lo que se escribe en metafísica, etc. Pero sería ocioso exigir a todos quienes escriben dogmática jurídica, filosofía práctica, metafísica, etc., refutar en cada trabajo al escéptico radical. Es mejor proceder como se hace normalmente: las disciplinas avanzan sobre supuestos, abiertas siempre al cuestionamiento de los mismos, de forma tal que el desarrollo de una disciplina no se detiene a esperar a que se persuada al último escéptico, ni el escéptico necesita esperar a que se acabe el flujo de academia constructiva para que su trabajo tenga un espacio.
}

${ }^{5}$ Ella es tratada así por autores con aproximaciones tan diferentes como Finnis (o el propio Fuller), Raz o Rawls. Hemos abordado este punto en Urbina y Contreras (2019). 
para "deseables") que un sistema de reglas debe satisfacer para poder ordenar la conducta humana en base a reglas. Dichas exigencias son: que las reglas sean generales, estén promulgadas, no sean retroactivas, sean claras, no contradictorias, no exijan lo imposible, constantes durante el tiempo, y que haya congruencia entre la acción de los funcionarios que aplican el derecho y regla declarada (Fuller, 1969).

En efecto, los desiderata constituyen ciertas cualidades de un sistema de normas jurídicas que permiten que éstas puedan efectivamente ordenar la conducta humana. Si las normas jurídicas, por ejemplo, son ininteligibles, entonces no pueden ser comprendidas por sus destinatarios y, por lo tanto, el derecho no podrá ordenar su conducta. Si ordenan lo imposible tampoco podrá hacerlo. Lo mismo puede decirse respecto a los demás desiderata. Nuestra tesis, que defenderemos en la siguiente sección, es que un desideratum adicional consiste en que el derecho ordene la conducta humana de forma directa.

Es importante precisar que en el esquema de Fuller el derecho no sólo ordena la conducta de los particulares. También la de todos los agentes que, de un modo u otro, aplican el derecho. Esto es, en efecto, de lo que se trata el último desideratum: la congruencia entre la regla declarada y la conducta de los funcionarios que la aplican (Fuller, 1969, p. 81-91). En este sentido, la ordenación de los segundos es una de las formas más relevantes en que el derecho permite la acción organizada de la comunidad. Así, por ejemplo, el derecho prohíbe a todas las personas cometer homicidio, y esta prohibición, en su sentido más grueso, aspira a ordenar la conducta con el fin de evitar que las personas cometan homicidios. Esto, a su vez, realiza una exigencia de la razón práctica — como se explicará en la tercera sección-: respetar la vida de otros y no quitar directamente la vida a un inocente. Pero esta regla está destinada también a quien la aplique. En conjunción con otras categorías jurídicas, la prohibición del homicidio pretende ordenar la conducta de jueces, fiscales, abogados, oficiales de la administración y otros que, al aplicar y operar con el derecho, ven su conducta (incluyendo su deliberación) en gran medida dirigida y, en este sentido, ordenada por el derecho que aplican (Webber et al., 2018). Es importante notar la complejidad de esta dirección jurídica dirigida primordialmente a quienes operan y aplican el derecho, que muchas veces es muy superior a la ofrecida por meras reglas primarias que prescriben directamente conductas fácilmente identificables que se deben realizar o no realizar (como "no virar izquierda"). Parte importante de la dirección que el derecho ofrece está dada por un complejo entramado de categorías jurídicas que apuntan a ordenar la forma en que los agentes jurídicos atribuirán significación jurídica a determinados hechos. Sólo de esta manera, y en conjunción con otras categorías, se podrá establecer una 
ordenación de la conducta ${ }^{6}$. Ellas cumplen un rol indispensable en la ordenación de la conducta humana en los altos niveles de complejidad en que opera el derecho.

\section{El derecho como forma directa de ordenar la conducta humana}

Siguiendo a Fuller, entonces, el derecho aspira a ordenar la conducta humana; y para hacerlo, debe satisfacer ciertas condiciones ("deseables") para lograrlo de forma adecuada. Los ocho desiderata son ejemplos de tales condiciones. En esta sección queremos justificar y explicar uno adicional: que el derecho debe ordenar la conducta de forma directa.

Existen diversas formas de ordenar la conducta humana. Aquí distinguimos dos: directa e indirecta. Se da una ordenación de forma directa cuando aquello que brinda la ordenación (un consejo, una norma, un conjunto de normas, etc.) significa $^{7}$ explícita o implícitamente el orden de la conducta esperado, de forma tal que si las personas desearan que su conducta se conformara con dicho orden podrían ver en la norma (consejo, etc.) en cuestión una guía para su conducta. Ejemplos de ordenación directa de la conducta son: una regla dada por el director de un hospital de no fumar en el recinto; o un consejo que un viejo médico da a todos sus pacientes de comer ajo y jengibre durante los meses de invierno. En estos casos la regla o el consejo significan el orden esperado (que no se fume en el hospital; que los pacientes consuman ajo y jengibre en invierno) y, así, al ser adoptadas como pautas de conducta, permiten al destinatario conformar su acción con el orden esperado. Por otro lado, se ordena la conducta humana indirectamente cuando aquello que la ordena (una directriz, una norma, un consejo, pero también un estímulo, un impedimento físico, etc.) no significa explícita o implícitamente el orden de la conducta esperado. Esta forma de dar un orden para la conducta admite, a su vez, dos tipos. A la primera la denominamos "comunicativa", y consiste precisamente en una comunicación: la transmisión de un mensaje ${ }^{8}$. Un ejemplo de esta forma tiene lugar en aquellos casos en que se aspira a ordenar la conducta mediante una comunicación, pero ella no significa la conducta esperada o deseada sino una distinta, bajo la expectativa de que por medio de ella será más probable que se lleve a cabo la conducta realmente deseada o esperada. Esto ocurre en lo que se denomina "psicología inversa". Así, por ejemplo, un padre da una orden a

\footnotetext{
${ }^{6}$ Sobre esto se puede decir lo que Fuller afirmaba de la expresión "código de conducta". Para él, dicha expresión indicaba no sólo un aspecto negativo, sino también incluye "el significado que confiere a acciones predecibles y aprobadas, las que luego proveen un punto de orientación para respuestas de interacción en curso [ongoing]" (Fuller, 1969 b, p. 3). Uno de nosotros ha analizado la complejidad de la dirección jurídica en mayor detalle en Urbina (2017).

${ }^{7}$ El verbo significar puede parecer, a primera vista, algo oscuro. Lo entendemos como la acción de indicar, dar a conocer, hacer saber, manifestar, declarar, designar, representar, ser un símbolo de, o mostrar, una idea u objeto. A lo largo del artículo usaremos el término significar en este sentido.

${ }^{8}$ Entendemos el término "comunicativo" en un sentido acotado, donde el mensaje que se transmite se hace de manera verbal. En un sentido amplio, el término también podría englobar lo que más abajo denominamos ordenación "fáctica".
} 
su hijo rebelde de beber algo de alcohol todas las semanas con la expectativa de que ello aumente las probabilidades de que su hijo no beba alcohol. En este caso, el estado de cosas que se desea lograr (que el hijo no consuma alcohol) es distinto del orden que la norma significa (que se consuma alcohol todas las semanas). Otra forma de ordenar indirectamente la conducta la denominamos "fáctica". Consiste en hacerlo por medio de estímulos ${ }^{9} \mathrm{o}$ impedimentos físicos. Ejemplos de esta forma de ordenar la conducta serían el caso de un anfitrión que apaga la calefacción de su hogar tras la cena para que sus invitados, motivados por el frío, se retiren a una hora prudente; o una municipalidad que instala resaltos en ciertas calles para que los vehículos que circulan por ahí disminuyan la velocidad. En ninguna de las formas indirectas puede el destinatario de la ordenación usarla directamente como una guía para conformar su conducta al orden deseado ${ }^{10}$.

Es deseable que el derecho ordene la conducta humana de la forma que aquí denominamos directa, mediante la proyección de un cierto orden de la conducta deseada ( $y$, al igual que en los demás desiderata de Fuller, el derecho típicamente opera así). Nótese, en primer lugar, que la satisfacción del desiderátum que aquí estamos presentando no requiere que la ordenación de la conducta por medio del derecho sea siempre realizada mediante imperativos ("no mate"), sino simplemente que dicha ordenación signifique el orden de conducta deseado, y no otro (o ninguno), de forma que los destinatarios puedan utilizarla como guía de su acción. De hecho, gran parte de las normas jurídicas no adoptan la forma imperativa, sino más bien disponen consecuencias jurídicas para ciertos hechos. Así, al identificar, en este tipo de casos, acciones que considera ilícitas, o los modos de llevar a cabo una determinada conducta para que produzca ciertos efectos jurídicos, el derecho significa un tipo de orden, que espera que sea realizado ${ }^{11}$. En este sentido, las prohibiciones penales, que comúnmente no adoptan la forma imperativa sino que

\footnotetext{
${ }^{9}$ En el sentido de un "agente físico, químico, mecánico, etc., que desencadena una reacción funcional en un organismo", en la primera acepción que da el diccionario de la RAE sobre la palabra "estímulo", como cuando hablamos de "estímulos sensoriales", y no en el sentido de "incentivo". Una ordenación directa pueda dar también incentivos para actuar, y las ordenaciones directas pueden ser analizadas en términos de incentivos (al estilo del law and economics) aun cuando dichos incentivos no agoten las razones para la acción que otorga el derecho. Asimismo, el hecho de que el derecho pueda recurrir a la coacción para asegurar su eficacia no lo convierte en una forma de ordenación indirecta de la conducta: la ordenación del derecho no se produce por estímulos ni mera manipulación física (por pura coacción), sino primariamente comunicando un cierto orden, aun cuando alguna infracción de los estándares que constituyen dicho orden pueda traer como consecuencia una medida de coacción. Para un estudio reciente de la relevancia de la coacción en la comprensión del derecho, véase Schauer (2015).

${ }^{10}$ Sí pueden usarlo como un antecedente en una especulación sobre los motivos de la ordenación, y sólo en ese sentido secundario se puede decir que una ordenación indirecta puede ser una "guía" para la conducta (unida a la disposición a adaptar la propia conducta a los deseos o expectativas del ordenante). Así, quien ve un resalto en la calle puede razonar que el propósito de dicho obstáculo es que uno reduzca su velocidad, y de esta forma identificar un orden de la conducta esperado; pero el resalto no es un estándar de conducta, ni (en nuestra cultura) la comunicación de dicho estándar. Es, simplemente, un obstáculo destinado a producir un efecto esperado (la reducción de la velocidad). Quien no reduce la velocidad al pasar por un resalto no vulnera ningún estándar, o al menos ningún estándar significado por dicho resalto.

${ }^{11}$ Así, Voegelin (1991) apunta que "las reglas jurídicas, sean reglas generales o reglas individuales para las partes en un caso concreto, tienen el carácter de proyectos de orden. Sea que la regla utilice la fórmula 'Harás' o 'No harás' o no lo haga, tiene ese significado cuando proyecta los tipos a los cuales la conducta de seres humanos se supone que se debe conformar" (p. 44).
} 
asocian una consecuencia jurídica a un hecho, comunican el rango de conductas criminalizadas. Es decir, conductas que los destinatarios de las normas deben entender como prohibidas. Al entender que ellas comunican acciones ilícitas que, por lo tanto, no se deben realizar, quien desee conformar su acción con el derecho puede ver en estas normas una guía para su acción. Más aun, quienes aplican el derecho pueden ver en el derecho penal una pauta para deliberar con mayor claridad $^{12}$. Lo mismo se puede decir de otras áreas o departamentos. Las normas del derecho civil comúnmente - junto con prohibir o mandar derechamente ciertas acciones- (i) señalan los modos en que ciertos actos jurídicos se deberían llevar a cabo para producir efectos jurídicos, (ii) determinan el significado jurídico de ciertos hechos jurídicos y actos jurídicos, e (iii) indican posibilidades jurídicas frente a ciertos hechos y las consecuencias (efectos) que ellos producirían. Todo esto implica una cierta ordenación de la conducta humana. Así, en (i) la conducta humana se ajusta a cierta forma para alcanzar ciertos resultados; en (ii) se atribuyen a ciertos hechos y actos un determinado significado jurídico (y esto en sí mismo es un orden —coordinación, específicamente-crucial de la conducta, como se verá más abajo) y se actúa en conformidad con esto; y en (iii) frente a ciertos hechos las personas realizan determinadas conductas consistentes con las posibilidades señaladas por el derecho, y de las opciones que tomen se seguirán determinadas consecuencias, con lo cual se genera un cierto orden de la conducta de distintas personas ${ }^{13}$. Asimismo, los jueces y otros agentes del derecho utilizarán las categorías del derecho civil al resolver disputas o solicitudes, asumiéndolas como guía para su deliberación y decisión. No es posible ni necesario mostrar exhaustivamente aquí cómo cada forma de operar del derecho significa un tipo de orden de la conducta humana que coincide con el orden deseado y sirve como guía para la acción. El objetivo, más bien, es ilustrar un punto más modesto: el desideratum de guiar la conducta humana de forma directa es compatible con una diversidad de técnicas para expresar las demandas del derecho, no sólo con la forma imperativa, sino con el amplio abanico de formas que expresan las normas jurídicas, también aquellas que se expresan en indicativo.

¿Cuál es la justificación de este desideratum? El que el derecho dirija la conducta humana de forma primordialmente directa parece obedecer a dos razones. Primero, el derecho ordena una enorme cantidad y diversidad de conductas en contextos altamente complejos. Como señala Finnis (2011b), "nada salvo un orden jurídico" puede proveer "un método imparcial de relacionar cargas con beneficios, y personas con personas, a través de un conjunto de personas y aspiraciones y

\footnotetext{
12 Para una explicación de la utilidad técnica de utilizar la forma indicativa, por ejemplo, en relación a conductas punibles, véase Finnis (2011a, p. 282-283).

${ }^{13}$ Ejemplo de esto último es el Art. 847 del Código Civil chileno: frente al hecho de que un predio no tenga acceso a los caminos públicos por encontrarse entre estos y aquel otros predios, el dueño del primero puede ejercer el derecho de imponer a los otros la servidumbre de tránsito pagando el valor del terreno necesario para la servidumbre y resarciendo perjuicios; la conducta de unos (que permitirán al menos el tránsito por sus predios) y de otros (que indemnizarán los perjuicios y pagaran el valor del terreno) es especificada por el derecho para ese tipo de situación, de forma tal que unos y otros pueden ver en éste una guía a su conducta.
} 
transacciones inmensamente complejo y duradero pero cambiante" (p. 65). La regulación del intercambio económico es un buen ejemplo. Las distintas formas para asociarse con un capital común con el fin de desarrollar una determinada actividad económica requiere una determinación autoritativa de cómo funcionará el régimen de responsabilidad de los socios (si personal o limitada, por ejemplo); o el modo de distribuir las utilidades; o la manera en que se ofrecerán las participaciones en el capital (las acciones) a terceros; o si los accionistas minoritarios tendrán formas de protección frente a las decisiones de los mayoritarios; o cómo funcionará el control de una sociedad(es) con otra(s), especialmente cuando se generan estructuras societarias en diversos países; etc. No parece posible ordenar la conducta humana a esta escala si no es por vía directa. En efecto, las distintas formas indirectas de ordenación, en general, se dan en contextos más bien simples, en la interacción de una persona o un grupo acotado de personas con otra persona o grupo acotado de personas, y respecto a una determinada conducta también bastante específica. Esto es así tanto para las formas "comunicativas" como las "fácticas", pero especialmente para las segundas. La razón consiste en que es ciertamente imposible regular la complejidad, variedad, y cantidad de los asuntos que el derecho debe coordinar — varios de ellos de naturaleza más bien abstracta (piénsese, por ejemplo, en la regulación del mercado financiero o de la propiedad intelectual) — por medios no "comunicativos" ( $y$, en particular, de forma no verbal). Con todo, las formas indirectas "comunicativas" también son insuficientes para llevar a cabo la tarea de coordinación de los asuntos humanos que realiza el derecho. Por cierto, esto no excluye que el derecho pueda regular, en alguna ocasión, la conducta humana por vía indirecta. Pero sí implica que esto es altamente excepcional, y para la regulación de asuntos más bien simples y altamente acotados.

Segundo, la ordenación de la conducta humana por vía indirecta es moralmente más problemática. Implica una medida de manipulación —particularmente en el caso de las formas "comunicativas" — en que la ordenación es, de algún modo, deliberadamente errónea. Tal vez esta forma de ordenar la conducta pueda ser aceptable en ciertos contextos, pero no en todos. El derecho está dirigido centralmente a adultos capaces de internalizar sus normas, de incorporarlas en su razonamiento práctico al deliberar y actuar. Se trata, por lo tanto, de un tipo de sujeto respecto del cual parece difícil justificar que los órganos creadores del derecho (autoridades legislativas, administrativas, y judiciales, por ejemplo) pretendan en general ordenar su acción sin proyectar de manera inteligible el orden conforme al cual pretenden que el agente conforme, deliberadamente, su conducta. En otras palabras, ordenar su conducta de manera indirecta. No es posible explicar acá en qué casos la forma indirecta de ordenar la conducta puede ser moralmente aceptable (si, por ejemplo, respecto de amigos o parientes resulta más aceptable que respecto de personas vinculadas sólo por un vínculo de ciudadanía o de regulador-regulado; o respecto de niños más que de adultos, etc.). Baste con advertir que es más problemática, y que parece ser moralmente inadecuada e ineficaz para la generalidad del derecho. 
Ambas razones conectan con aspectos centrales de la teoría de Fuller. En efecto, respecto de la primera razón, ordenar la conducta humana es una exigencia del cometido que el derecho pretende cumplir. En los términos de Fuller, someter la conducta humana al gobierno de las reglas supone la aptitud de ordenarla. Y hemos explicado que el que la ordenación sea directa es esencial para que las reglas sean aptas para ordenar la conducta humana en los niveles de complejidad y abstracción en que opera el derecho. Respecto de la segunda razón, se debe enfatizar un aspecto destacado recientemente por Rundle (2012, p. 97-101): que para Fuller la teoría de los desiderata responde a una "visión del hombre" que se haya "implícita" en el propio sistema de los desiderata. Si bien las exigencias necesarias para que el gobierno de las reglas sea posible es, en el grueso, "neutral" respecto de lo que podríamos denominar "fines" del derecho (o "moral externa", en el lenguaje de Fuller), Fuller argumenta que dichas exigencias implican, necesariamente, un compromiso con la idea de que el destinatario de la norma es alguien con capacidad de agencia: que pueda entender y actuar conforme a lo que entiende. En otras palabras, que pueda aprehender la ordenación que hace el derecho mediante normas jurídicas, incorporándolas en su razonamiento práctico. Sólo así, sostiene Fuller, es que el derecho puede tratar al hombre con dignidad (Fuller, 1969, p. 162-167). Por el contrario, intentar ordenar su conducta mediante estímulos o manipulación - i.e. indirectamente- es una manera de instrumentalizar al destinatario de la norma. Aquí es donde Fuller distingue al derecho de otra forma de ordenar la conducta humana. El derecho, explica, no es lo mismo que la "dirección administrativa" [managerial direction]. Aunque ambas implican la dirección y control de la actividad humana y la subordinación a una autoridad, el derecho tiene una cualidad esencial que las diferencia. Las normas jurídicas están ideadas y diseñadas para que el destinatario pueda seguirlas (lo que implica, como hemos explicado, usarlas como razón para la acción, incorporándolas en su razonamiento práctico), y ellas no sólo pretenden guiar la conducta de un solo individuo o del individuo con la autoridad generativa de la norma, sino que $-y$ principalmente - sirven el propósito de establecer y determinar las relaciones entre individuos, para coordinarlas. Las directrices que emanan de un gerente o principal, en cambio, están destinadas para ser aplicadas por el subordinado de modo de servir al propósito fijado por el superior de la dirección administrativa y no el de sus propios asuntos; y son normalmente particulares, sin afán de pretender coordinar al resto de los subordinados (Fuller, 1969, p. 207-210; véase ahí, en particular, las tensiones entre la dirección administrativa y los ocho desiderata).

Si el derecho, entonces, ha de ordenar la conducta humana de forma directa, debe guiarla, y para ello debe proveer dirección jurídica. Entendemos "dirección jurídica" del siguiente modo. Recordemos que las formas directas de ordenar la conducta humana están orientadas a que la ordenación sea incorporada en el razonamiento práctico del destinatario de la norma, de forma tal que la asuma 
como una razón para adoptar una determinada conducta ${ }^{14}$. Así, por ejemplo, una norma que dispone "detenga el auto en la luz roja" es entendida por el destinatario de la norma como una razón para detener el auto en una luz roja. En el caso del derecho, se trata de una forma de ordenar la conducta humana que es autoritativa $y$, por tanto, pretende proveer de una razón concluyente, al menos en el grueso de los $\operatorname{casos}^{15}$. Al establecer un curso de acciones o de omisiones que se ha de realizar, el derecho, como toda forma directa de ordenar la conducta humana, lo hace por la vía de informar la deliberación de aquellos a quienes está dirigido. Esto, que es típicamente implícito (pero no por ello menos real) en el caso del ciudadano, es muy patente en el caso de los oficiales del derecho, cuya tarea es aplicarlo a situaciones concretas y que (a diferencia de los ciudadanos) tienen la carga de justificar jurídicamente y de forma verbal su decisión. Este es el caso de jueces y órganos administrativos que resuelven reclamos $u$ otros asuntos de naturaleza jurisdiccional o similares.

En suma, si bien es cierto que el derecho ordena la conducta humana, no lo hace, típicamente, de cualquier modo. No ordena la conducta mediante manipulación o estímulos, sino de la forma que aquí he denominado "directa", es decir, significando el orden deseado para que éste sea aprehendido por quienes someten su acción al derecho. La manera de hacerlo es mediante lo que hemos denominado dirección jurídica.

\section{La ordenación de la conducta humana que hace el derecho está orientada a satisfacer ciertas exigencias de la razón práctica}

En la sección anterior explicamos el desideratum de la ordenación directa de la conducta por parte del derecho, y ofrecimos dos razones para justificarlo. Conviene ahora ahondar en la explicación del desideratum, explorando con algún detalle mayor cómo el derecho ordena la conducta de forma directa.

Para ello debemos introducir un postulado adicional. El derecho no es un orden arbitrario. Está orientado a realizar ciertas exigencias de la razón práctica, es decir, exigencias relativas a cómo deliberar y obrar bien ${ }^{16}$. Esto no implica asumir una tesis controversial respecto de la naturaleza del derecho y sobre si la moralidad es

\footnotetext{
${ }^{14}$ La comprensión del derecho como otorgando "razones para la acción" es también común a diversas teorías del derecho, y su tratamiento canónico se encuentra tanto en teorías iuspositivistas como iusnaturalistas prominentes. Así, véase por ejemplo Hart (2012), Raz (1999), Finnis (2007).

${ }^{15}$ Acaso por la vía de proveer razones "exclusionarias", es decir, razones que excluyen a una serie de razones rivales (por ejemplo, la mera conveniencia para el sujeto de derecho de adoptar un curso de acción alternativo al exigido por el derecho) para cada caso regulado. Sobre razones "exclusionarias" véase Raz (1999, especialmente p. 35-48 y 178-199).

${ }^{16} \mathrm{Al}$ hablar de exigencias relativas a deliberar y obrar bien nos remitimos a una categoría amplia, que comprende exigencias de justicia, morales, políticas, de eficiencia, etc., en su relación más razonable.
} 
parte del contenido o de los criterios de validez del derecho. No es necesario, para nuestros efectos, postular que exista alguna relación de este tipo. Pero tampoco es necesario excluir esta visión. Ambas posibilidades pueden ser compatibles con el supuesto formulado acá y con la tesis general del artículo. Baste con asumir que, aun cuando el derecho sea un orden técnico y artificial, está orientado a la satisfacción de exigencias morales, políticas, prudenciales, etc. Así, se puede decir que el derecho, en tanto orden social, intenta "hacer que [los seres humanos] se abstengan de determinados actos que por alguna razón se consideran perjudiciales a la sociedad, y que realicen otros que por alguna razón repútanse útiles a la misma" (Kelsen, 1949, p. 15). O que "un impulso que es una parte viva de incluso el sistema jurídico más equivocado y arbitrario es un impulso [...] de hacer que el sistema, sus detalles y sus agentes, estén más cerca de realizar un ideal de justicia" (Llewelyn, 2008, p. xxviii-xxvix). O, en términos más generales, se puede afirmar que el derecho intenta "maximizar conformidad con razones subyacentes" (Raz, 1999, p. 193-194). Es, en este sentido, un orden técnico al servicio de un orden moral (Finnis, 1990). Solo tiene sentido que haya derecho si éste responde a una exigencia de la razón práctica, es decir, que haya buenas razones (de justicia, políticas, económicas, etc.) para tenerlo ${ }^{17}$. Así, el punto es muy simple y no controvertido, como lo demuestra el amplio catálogo de escuelas ejemplificadas por los autores recién citados (positivistas, realistas jurídicos, iusnaturalistas, entre otros): el sistema jurídico — su forma y parte medular de su contenido— no es arbitrario, tiene una razón de ser en virtud de su capacidad de realizar diversas exigencias morales, prudenciales y, típicamente, exigencias de justicia.

El vínculo entre este supuesto y la idea de que el derecho ordena la conducta humana de forma directa es de la mayor importancia. Dado que la ordenación de la conducta humana que efectúa el derecho está orientada a la realización de exigencias de la razón práctica, y que el derecho ha de ordenar la conducta humana de forma directa, podemos articular el vínculo entre ambas ideas: el derecho expresará las exigencias de la razón práctica de manera tal de poder ordenar, en consonancia con ellas, la conducta humana de forma directa. Lo hará de modo que permita a los sujetos del derecho aprehender esas exigencias e incorporarlas a su propio razonamiento práctico, guiando, así, su actuar. La expresión del orden jurídico es la expresión de las exigencias que lo constituyen.

Es útil aclarar cuatro aspectos de esta idea. Primero, la expresión "exigencias de la razón práctica" es convenientemente ambigua. Ellas pueden referirse a conductas específicas o genéricas, o a consideraciones para evaluarlas. Así, pueden formularse como conductas específicas que la razón práctica determina aquello se ha de hacer o abstenerse de hacer en ciertos casos (es decir, que hay una exigencia racional

\footnotetext{
${ }^{17}$ Esta idea es desarrollada especialmente en Finnis (1990, p. 4-7). Para Shapiro, de forma similar, el "objetivo fundamental del derecho es satisfacer [una] demanda moral" que consiste en superar ciertas deficiencias de la vida social que Shapiro asocia a las "circunstancias de la legalidad" (Shapiro, 2011, p. 213).
} 
de hacerlas o no hacerlas bajo ciertas condiciones; por ejemplo, que no se debe adelantar a otro vehículo en una curva). También pueden formularse de modo más general (que se debe conducir a una velocidad prudente). Y también pueden formularse como consideraciones relevantes para evaluar una conducta (al evaluar el nivel de responsabilidad del conductor en un daño que haya causado, se pondrá atención a la intención con que obró en causarlo). Por supuesto, es posible que una exigencia que esté formulada en términos de una conducta como un estándar para evaluar una conducta puede, desde otro punto de vista, verse como una exigencia de realizar una conducta general o precisa.

El punto acá es simplemente llamar la atención respecto a que el derecho expresa exigencias de la razón práctica de distintas formas, en algunos casos dando expresión a consideraciones para la evaluación de cursos de conducta, en otros expresando conductas específicas de lo que se debe o no hacer. Así es como se aparece, plásticamente, el conjunto de normas jurídicas a quien aplica y se somete al derecho ${ }^{18}$.

Segundo, el derecho ordena la conducta humana no sólo mediante prescripciones, sino también mediante categorías jurídicas que, en principio, no parecen directamente prescriptivas, tales como definiciones, requisitos, condiciones, etc., todas las cuales inciden en la determinación del significado jurídico de los hechos a los que aplican, lo cual, en último término, determina el contenido de la ordenación que el derecho hace.

Estas categorías jurídicas también responden a exigencias de la razón práctica. Así, un juez que ve un caso de homicidio presunto cuenta con una serie de categorías jurídicas para hacer sentido del caso y, específicamente, para determinar si cierta conducta puede ser entendida como "homicidio" para los efectos del derecho. Estas categorías no son arbitrarias, sino que intentan capturar consideraciones relevantes sobre la justicia desde la perspectiva de la reacción penal. Por ejemplo, los elementos del delito (incluyendo la descripción de la conducta típica, las causales de justificación, eximentes de responsabilidad, entre otros) contenidos en el ordenamiento jurídico en categorías jurídicas específicas, expresan consideraciones morales relevantes para el tipo de casos en que aplican. Así, la distinción entre dolo y culpa (contenida en las categorías jurídicas referidas al tipo subjetivo) apunta a la idea de que es injusto castigar un hecho punible que se realiza con, digamos, dolo directo, que otro que es igual en todo salvo en ser realizado

\footnotetext{
${ }^{18}$ En oposición a la perspectiva del teórico que provee una sistematización de esa experiencia jurídica inmediata de quien aplica o se somete al derecho. Acá nos interesa cómo el derecho ordena la conducta humana de forma principalmente directa, y esto lo hace en la forma en que éste se aparece a quienes lo aplican y se someten a él, y no en la forma en que (algún aspecto de él) puede ser expresado tras la reconstrucción del teórico —a menos que la reconstrucción del teórico se convierta en canónica, en cuyo caso pasa a formar parte del derecho (pero no es el caso en la distinción mencionada más arriba)—.
} 
simplemente con culpa ${ }^{19}$. En otras palabras, existe una diferencia moral significativa entre intentar algo malo y producirlo como efecto secundario (deseado o no) de lo que se intenta. Lo primero es más reprochable que lo segundo. El derecho, al contener esta distinción y darle expresión mediante categorías jurídicas, ordena la conducta del juez, dirigiéndolo a confrontar si en el caso que juzga la conducta en cuestión fue dolosa o culposa. De este modo, las categorías jurídicas que, en principio, no parecen prescriptivas no son por ello arbitrarias, sino que también pretenden responder a exigencias de la razón práctica. En esto el derecho no se distingue de otras disciplinas, en que para abordar un ámbito de la realidad de manera sofisticada se requiere de un aparataje conceptual elaborado que sea más preciso que los conceptos posibles de transmitir mediante los términos del lenguaje ordinario $^{20}$. Un aparataje conceptual tal es provisto por innumerables categorías jurídicas que permiten expresar de forma más exacta que el lenguaje común exigencias de la razón práctica y, por tanto, las formas de ordenar la conducta de modo directo que éstas proponen en las diversas situaciones.

Tercero, dado que en muchas áreas es necesario que haya una determinación autoritativa de un asunto que está sub-determinado por la razón, las categorías jurídicas que dan expresión a una determinación tal, también están orientadas a realizar exigencias de la razón práctica —comúnmente, la exigencia de que para cierta área haya una decisión autoritativa- ${ }^{21}$. Así, por ejemplo, un sistema de tributación debe satisfacer, entre otras, ciertas exigencias de justicia distributiva. Una exigencia de este tipo puede demandar que aquellos que poseen más colaboren en una mayor proporción con el financiamiento del Estado y su actividad. Ahora, cuál debe ser exactamente la escala de progresión de los impuestos es algo que la razón práctica no puede establecer con absoluta precisión. Y, sin embargo, sí puede disponer que es necesario que exista una escala precisa: sin ella, el sistema tributario no puede ser eficaz, y no siéndolo, no se podrán satisfacer ciertas exigencias morales y políticas — como aquellas de justicia distributiva que demandan que quienes tienen más riqueza colaboren en mayor proporción con el financiamiento de los asuntos públicos - que la razón práctica puede identificar como relevantes y como aptas de ser satisfechas por un sistema tributario. Dada la necesidad de que exista una escala precisa, se requiere, asimismo, que la autoridad competente determine esa escala. Al hacerlo por medio del derecho, se ordena la conducta de agentes del sistema tributario y de contribuyentes de forma eficaz, y las categorías jurídicas de esa esfera están orientadas a la realización de las exigencias de la razón práctica recién mencionadas: la exigencia de justicia de que quienes tienen más paguen más impuestos, y la exigencia que un sistema tributario establezca una escala de progresión de impuestos precisa de manera eficaz. Si bien estas no son las únicas exigencias vinculadas a un sistema de tributación, permiten ilustrar

\footnotetext{
${ }^{19}$ Para una explicación de este asunto, ver Girgis (2013).

${ }^{20}$ Debemos a Andrés Peñaloza esta observación.

${ }^{21}$ Sobre esto, véase la idea de determinatio en Finnis (2011a, p. 284-286).
} 
cómo las categorías jurídicas están orientadas a dar expresión a distintas exigencias de la razón práctica, y también aquellas que expresan una determinación de la autoridad en un asunto que no está completamente determinado por ella.

Y cuarto, el conjunto de exigencias de la razón práctica que el derecho realiza en cualquiera de sus áreas es altamente complejo. No se trata de una sola exigencia, sino de una multiplicidad de ellas; no de un solo tipo (por ejemplo, exigencias de justicia) sino de varios (de justicia, de eficacia, de eficiencia, de prudencia política, etc.); que no exigen una cosa (que no se haga algo) sino varias distintas (que algo se haga o no se haga, que a algo se le atribuya un cierto efecto, que algo sea posible, etc. $)^{22}$; y que aplican a situaciones muy diversas donde, consecuentemente, el conjunto de exigencias varía según la situación. Todo esto repercute en el derecho. Para poder ordenar la conducta de modo directo adecuadamente en los diversos ámbitos en que aplica, debe recoger esta complejidad, y para ello debe contar con un conjunto de conceptos de similar (alta) complejidad —una multiplicidad de categorías jurídicas precisas, específicas para distintas situaciones, y, por lo tanto, diferenciadas - . Esta complejidad de las categorías jurídicas, sensibles a las diversas exigencias de la razón práctica que constituyen el complejo orden deseado por el derecho moderno, le permiten expresarlas y, así, guiar la conducta de acuerdo con ellas, es decir, ordenándola de forma directa ${ }^{23}$.

\section{Conclusión}

Se acepta ampliamente que el derecho pretende ordenar la conducta humana. Avanzar en una caracterización más completa de cómo el derecho la ordena exige reconocer otro aspecto elemental de la forma en que opera el derecho, a saber, que el derecho no ha de ordenar la conducta de cualquier modo, sino principalmente de modo directo. Así, no lo hace mediante estímulos o manipulación sino primordialmente significando el orden deseado. Hemos ofrecido dos razones fundamentales para justificar esto. Primero, dado que el derecho suele operar en contextos altamente complejos, la ordenación directa se presenta como un medio más eficaz que los medios indirectos. Segundo, la ordenación indirecta de la conducta es más problemática moralmente, pues implica una medida de manipulación. La ordenación directa, en cambio, al comunicar el orden deseado de conducta, supone que el destinatario de la norma es capaz de internalizarla e incorporarla en su razonamiento práctica al deliberar y actuar, respetando mejor su condición de agente moral.

\footnotetext{
${ }^{22}$ Nuevamente, no interesa aquí si es posible reducir lógicamente estas distintas exigencias a un solo tipo de exigencias o a un conjunto más acotado de tipos, o unas a otras. Aquí nada se gana con esta reducción.

${ }^{23}$ Aquí se encuentra el fundamento de la explicación de la complejidad del derecho positivo. Abordamos esta cuestión en mayor detalle en Urbina y Contreras (2019).
} 
Finalmente, postular la existencia de este desideratum adicional conecta con la noción ampliamente compartida de que el derecho busca satisfacer exigencias de la razón práctica (de justicia, morales, políticas, de eficiencia, etc.). Así, al ordenar la conducta de modo directo, el derecho comunica al ciudadano aquellas exigencias de la razón práctica que las reglas buscan satisfacer, de modo que el ciudadano las incorpore en su propio razonamiento, aprehendiéndolas y actuando conforme a ellas. De este modo, el derecho ofrece una genuina guía a la conducta humana. Esto constituye un desideratum de un ordenamiento jurídico en forma, junto a los demás desiderata tematizados por Lon Fuller.

\section{Referencias bibliográficas}

Alexy, R. (2007). Teoría de los derechos fundamentales. Madrid, España: Centro de Estudios Políticos y Constitucionales.

Austin, J. (1998). The Province of Jurisprudence Determined. Indianapolis, USA: Hackett.

Dworkin, R. (1978). Taking Rights Seriously. Cambridge, USA: Harvard University Press.

Dworkin, R. (1986). Law's Empire. Cambridge, USA: Harvard University Press.

Finnis, J. (1990). Natural Law and Legal Reasoning. Cleveland State Law Review, 38, 1-13.

Finnis, J. (2011a). Natural Law and Natural Rights. Oxford, UK: Oxford University Press.

Finnis, J. (2011b). Collected Essays of John Finnis, Tomo IV. Oxford, UK: Oxford University Press.

Fisher III, W.W., Horwitz, M.J. \& Reed, T.A. (1993). American Legal Realism. Nueva York, USA: Oxford University Press.

Fuller, L. (1969a). The Morality of Law. New Haven, USA: Yale University Press.

Fuller, L. (1969b). Human Interaction and the Law. American Journal of Jurisprudence, 14 (6), 1-36.

Girgis, S. (2013). The Mens Rea of Accomplice Liability: Supporting Intentions. Yale Law Journal, 123 , 460-494.

Grey, T.C. (1983). Langdell's Orthodoxy. University of Pittsburg Law Review, 45, 1-53.

Hart, H.L.A. (2012). The Concept of Law. Oxford, UK: Oxford University Press.

Jansen, N. (2010). The Making of Legal Authority. Oxford, UK: Oxford University Press.

Kelsen, H. (1979). Teoría pura del derecho. Ciudad de México, México: Universidad Nacional Autónoma de México.

Kelsen, H. (1949). Teoría general del derecho y del Estado. Ciudad de México, México: Imprenta Universitaria.

Llewellyn, K.N. (2008). The Bramble Bush. The Classic Lectures on the Law and the Law School. Nueva York, USA: Oxford University Press.

Llewellyn, K.N. \& Schauer, F. (Eds) (2011). The Theory of Rules. Chicago, USA: The University of Chicago Press.

Raz, J. (1999). Practical Reason and Norms. Oxford, UK: Oxford University Press.

Rundle, K. (2012). Forms Liberate: Reclaiming the Jurisprudence of Lon Fuller. Oxford, UK: Hart Publishing.

Schauer, F. (2015). The Force of the Law. Cambridge, USA: Harvard University Press.

Shapiro, S. (2011). Legality. Cambridge, USA: Harvard University Press.

Unger, R.M. (2015). The Critical Legal Studies Movement. Another Time, A Greater Task. Nueva York, USA: Verso. 
Urbina, F.J. (2017). A Critique of Proportionality and Balancing. Cambridge, UK: Cambridge University Press.

Urbina, F.J. y Contreras, F. (2019). La claridad como especificidad: una revisión del desideratum de la claridad en la teoría de Lon Fuller. Vniversitas 136 [en prensa].

Voegelin, E., Pascal, R.A., Babin, J.L., \& Corrington, J.W. (Eds.). (1991). The Nature of the Law and Related Legal Writings. Baton Rouge, USA: Louisiana University Press.

Webber, G., Yowell, P., Ekins, R., Köpcke, M., Miller, B.W., \& Urbina F. (2018). Legislated Rights: Securing Human Rights through Legislation. Cambridge, UK: Cambridge University Press. 